Control and data acquisition software upgrade for JET gamma-ray diagnostics

Santos, B.

Elsevier

2018

Santos , B , JET Contributors \& Ahlgren , T 2018 , Control and data acquisition software upgrade for JET gamma-ray diagnostics . in Fusion Engineering and Design . Fusion Engineering and Design , vol. 128 , Elsevier , Amsterdam , pp. 117-121 , IAEA Technical Meeting on Control, Data Acquisition, and Remote Participation for Fusion Research , Greifswald , Germany , 08/05/2017 . https://doi.org/10.1016/j.fusengdes.2018.01.064

http://hdl.handle.net/10138/326691

https://doi.org/10.1016/j.fusengdes.2018.01.064

unspecified

submittedVersion

Downloaded from Helda, University of Helsinki institutional repository.

This is an electronic reprint of the original article.

This reprint may differ from the original in pagination and typographic detail.

Please cite the original version. 
WPJET4-CPR(17) 17184

B. Santos et al.

\section{Control and Data Acquisition Software Upgrade for JET Gamma-Ray Diagnostics}

Preprint of Paper to be submitted for publication in Proceeding of 11th IAEA Technical Meeting on Control, Data Acquisition, and Remote Participation for Fusion Research

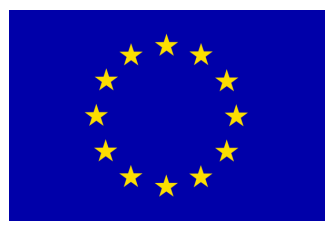

This work has been carried out within the framework of the EUROfusion Consortium and has received funding from the Euratom research and training programme 2014-2018 under grant agreement No 633053 . The views and opinions expressed herein do not necessarily reflect those of the European Commission. 
This document is intended for publication in the open literature. It is made available on the clear understanding that it may not be further circulated and extracts or references may not be published prior to publication of the original when applicable, or without the consent of the Publications Officer, EUROfusion Programme Management Unit, Culham Science Centre, Abingdon, Oxon, OX14 3DB, UK or e-mail Publications.Officer@euro-fusion.org

Enquiries about Copyright and reproduction should be addressed to the Publications Officer, EUROfusion Programme Management Unit, Culham Science Centre, Abingdon, Oxon, OX14 3DB, UK or e-mail Publications.Officer@euro-fusion.org

The contents of this preprint and all other EUROfusion Preprints, Reports and Conference Papers are available to view online free at http://www.euro-fusionscipub.org. This site has full search facilities and e-mail alert options. In the JET specific papers the diagrams contained within the PDFs on this site are hyperlinked 


\section{Control and Data Acquisition Software Upgrade for JET Gamma-Ray Diagnostics}

B. Santos ${ }^{\mathrm{a}}$, A. Fernandes ${ }^{\mathrm{a}}$, R.C. Pereira ${ }^{\mathrm{a}}$, A. Neto ${ }^{\mathrm{b}}$, J. Bielecki ${ }^{\mathrm{c}, \mathrm{d}}$, T. Craciunescu ${ }^{\mathrm{e}}$, J. Figueiredo ${ }^{\mathrm{a}, \mathrm{d}}$, V. Kiptily ${ }^{\mathrm{d}}$, A. Murari ${ }^{\mathrm{d}}$, M. Nocente ${ }^{\mathrm{f}, \mathrm{g}}$, D. Rigamontif ${ }^{\mathrm{fg}}$, J. Sousa ${ }^{\mathrm{a}}$, M. Tardocchi ${ }^{\mathrm{g}}$, L. Giacomelli ${ }^{\mathrm{g}}$, I. Zychor ${ }^{\mathrm{h}}$, A. Broslawski ${ }^{\mathrm{h}}$, M. Gosk ${ }^{\mathrm{h}}$, S. Korolczuk $^{\mathrm{h}}$, A. Urban ${ }^{\mathrm{h}}$, G. Boltruczyk ${ }^{\mathrm{h}}$, C.M.B.A. Correia ${ }^{\mathrm{i}}$, B. Gonçalves ${ }^{\mathrm{a}}$ and JET Contributors*

EUROfusion Consortium, JET, Culham Science Centre, Abingdon, OX14 3DB, UK

${ }^{a}$ Instituto de Plasmas e Fusão Nuclear, Instituto Superior Técnico, Universidade de Lisboa, 1049-001 Lisboa, Portugal

${ }^{b}$ Fusion for Energy, Josep Pla 2, 08019 Barcelona, Spain

'Institute of Nuclear Physics Polish Academy of Sciences, PL-31342 Krakow, Poland

${ }^{d}$ Culham Centre for Fusion Energy, Culham, United Kingdom

'Institute of Atomic Physics, Magurele, Ilfov, Romania

${ }^{f}$ Dipartimento di Fisica “G. Occhialini”, Università degli Studi di Milano-Bicocca, Milano, Italy

${ }^{g}$ Istituto di Fisica del Plasma "P. Caldirola", CNR, Milano, Italy

${ }^{h}$ Narodowe Centrum Badań Jadrowych (NCBJ), 05-400 Otwock, Poland

${ }^{i}$ LIBPhys-UC, Department of Physics, University of Coimbra, P-3004 516 Coimbra, Portugal

*See the author list of "Overview of the JET results in support to ITER" by X. Litaudon et al. to be published in Nuclear Fusion Special issue: overview and summary reports from the 26th Fusion Energy Conference (Kyoto, Japan, 17-22

October 2016)

The Joint European Torus (JET), the largest magnetic confinement plasma physics experiment in operation, has a large amount of key diagnostics for physics exploration and machine operation, which include several GammaRay Diagnostics.

The Gamma-Ray Spectrometer (GRS), Gamma Camera (GC) and Gamma-Ray Spectrometer Upgrade (GSU) diagnostics have similar Control and Data Acquisition Systems (CDAQs) based on the Advanced Telecommunication Computing Architecture (ATCA) standard, featuring Field Programmable Gate Arrays (FPGAs) for data processing and management. The installed ATCA CDAQ digitizer boards are connected to the host controller through its Peripheral Component Interconnect Express (PCIe) interface with dedicated in-house developed software drivers.

During past JET-EP2 enhancements, the GRS and GC diagnostics were successfully installed and commissioned. However, the installed CDAQ software that interfaces these diagnostics to JET COntrol and Data Acquisition System (CODAS) is different, requiring higher maintenance costs. While the GRS was implemented using FireSignal, GC used Multi-threaded Application Real-Time executor (MARTe) framework.

Benefiting from the Gamma Camera Upgrade (GCU) and new GSU installation and commissioning, the upgrading of the software and controller hardware used in the GRS and GC was evaluated, aiming at software standardization between all three diagnostics for easier maintenance. The MARTe framework was selected as CDAQ software and Scientific Linux as Operating System (OS).

This paper describes the software standardization process between the diagnostics towards the usage of the same CDAQ software as well as the same OS for the controllers, which allows the operator to minimize the maintenance time, avoiding the need for system specific expertise.

Keywords: Control, Data Acquisition, Software, MARTe, JET, Nuclear Fusion

\section{Introduction}

The Joint European Torus (JET) gamma-ray diagnostics are in the scope of the diagnostic package of the JET deuterium-tritium (DT) campaign in preparation for ITER [[1],[2]]. This includes the Gamma-Ray Spectrometer (GRS), Gamma Camera Upgrade (GCU) and Gamma-Ray Spectrometer Upgrade (GSU) projects.

The GRS (KM6S spectrometer) and GSU (KM6T spectrometer) projects aim at performing high-resolution gamma-ray spectroscopy at very high count rate while the GCU (KN3G camera) project is expected to improve the spectroscopic properties of the existing gamma-ray/hard $\mathrm{X}$-ray camera, in terms of energy resolution and high count rate capability, for DT campaign [[3]-[8]].

The GRS and the old version of GCU, the Gamma Camera (GC), were successfully installed and commissioned during past JET-EP2 enhancements. These systems are based on the Advanced Telecommunications Computing Architecture (ATCA) standard [9], which was selected for ITER fast controllers requiring demanding tasks (diagnostics, I\&C and Interlock) like plasma control system [[10],[11]]. 
The ATCA Control and Data Acquisition (CDAQ) system includes high frequency and reconfigurable digitizer modules (ATCA-TRP-400) [12] with embedded Field Programmable Gate Array (FPGA) devices, connected to the host controller (ATCA-CONTROLLERPCIe) [13] using the Peripheral Component Interconnect Express (PCIe) interface [14].

Both diagnostics have similar hardware, however, due to historical reasons, the installed CDAQ software that interfaces to JET COntrol and Data Acquisition System (CODAS) is different. While the GRS was implemented using FireSignal [[15],[16]], the GC uses the Multithreaded Application Real-Time executor (MARTe) framework [[17],[18]], which may lead to maintenance problems in the future.

The recent upgrade in the JET gamma-ray diagnostics comprised the new GSU installation and commissioning, and the GC upgrade and contributes to the study of a new solution, aiming at the usage of an updated host hardware (Motherboard, CPU, Memory and Hard-Drive) and software (Operation System and CDAQ software). To fulfill these requirements was designed the present solution, which contributes to reduce the system specific expertise and minimize maintenance time.

\section{Hardware environment}

Fig. 1 depicts the hardware environment of the installed systems.

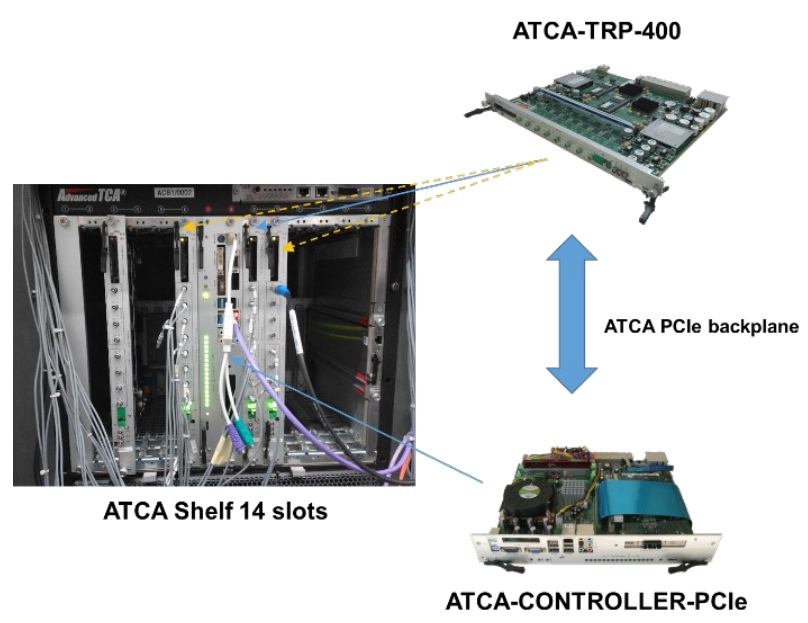

Fig. 1- Hardware Environment

\subsection{ATCA-TRP-400}

The ATCA-TRP-400 board was designed to perform acquisitions at high rates while featuring processing capabilities to discriminate only the relevant events. It is compliant with ATCA PICMG 3.0 specification [9] and comprises two blocks of FPGAs for data path and real time processing connected to 4 analog inputs of 13/14-bit

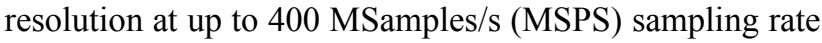
and $2 \mathrm{~GB}$ of DDR2 memory to store data. Furthermore, this module is connected to the host using the available $\mathrm{x} 1$ PCIe link [[3],[5],[12]].

\subsection{ATCA-CONTROLLER-PCIe}

The ATCA-CONTROLLER-PCIe host controller is compliant with the ATCA PICMG 3.0 specification [9]. The design is based on a PCIe switch connected to the ATCA full-mesh backplane and to micro Advanced Technology Extended (ATX) motherboard with an Intel ${ }^{\circledR}$ Core $^{\mathrm{TM}}$ i3-4170 Processor, 4GB DDR3 and 500 GB HD [13].

\subsection{ATCA Chassis}

The ATCA Chassis has 14 slots, with redundant shelf managers. All (three) diagnostics have installed similar host controller connected to the digitizers boards through the ATCA PCIe backplane.

\section{CDAQ System Uniformization}

During the JET-EP2 enhancements the GRS and GC diagnostics were successfully installed and commissioned using Firesignal as CDAQ Software [16] and Linux Fedora 8 as Operating System (OS), interfacing to the hardware with dedicated in-house developed software drivers. However, some years later, during the GC diagnostic enhancement for development of the real-time processing system to the JET hard X-Ray and GammaRay Profile monitor [6], the GC CDAQ software was upgraded to MARTe [17], keeping the same operating system and Linux Device Driver compatibles with MARTe, avoiding the replacement of a stable and tested version without valuable benefit at the time.

Benefiting from the GCU and new GSU installation and commissioning, the upgrading of the software and controller hardware used in the GRS and GC was evaluated, aiming at software standardization between the two existent diagnostics and the new diagnostic to install, once keeping three different solutions are hard to maintain.

Concerning the controller hardware, the proposed solution was the upgrade of the motherboard, CPU, memory and hard-drive to an up to date solution detailed in previous section, replacing the old Intel ${ }^{\circledR}$ Quad-Core CPU, 2 GB of DDR2 memory and 320 GB HDD [13]. 


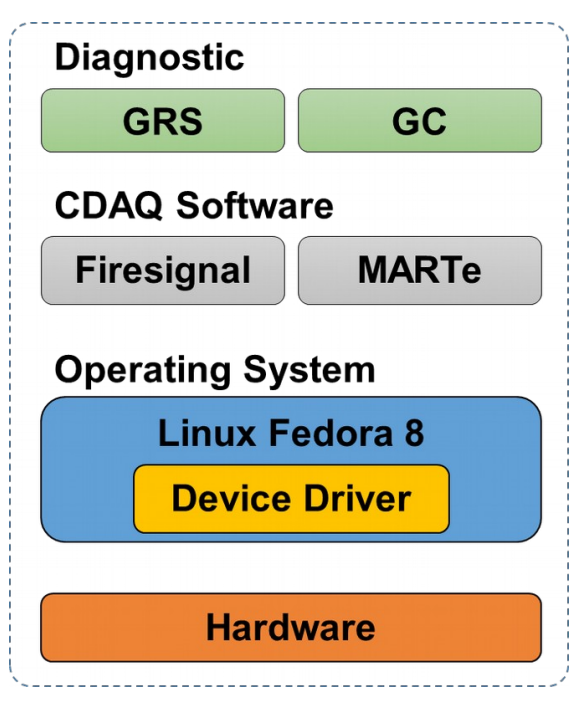

Fig. 2 - Existing Software Architecture

Fig. 2 depicts the software architecture of the existent systems, which is not fully compatible with new installed hardware, since Fedora 8 is no longer compatible with recent motherboards.

Concerning the software update, it was studied the uniformization between the three systems to use a transversal solution. The Scientific Linux 6.7 was selected as OS, since it is supported by JET CODAS, making the compatibility with other installed systems, forcing the Linux Device Driver upgrade to the new kernel version. For the CDAQ software, MARTe framework was selected because: (i) it fulfills all the diagnostics requirements; (ii) it is used in other JET diagnostics; (iii) it is popular in the fusion community by its usage in several machines, which contributes for its continuous development [[19]-[25]]. Fig. 3 depicts the new software architecture using Scientific Linux as OS and MARTe as CDAQ Software which includes the MARTe core, the CODAS Interface Library to connect the diagnostics to CODAS, the developed GAMs and IOGAMs for data acquisition and processing, the configuration files and startup files.
CDAQ Software
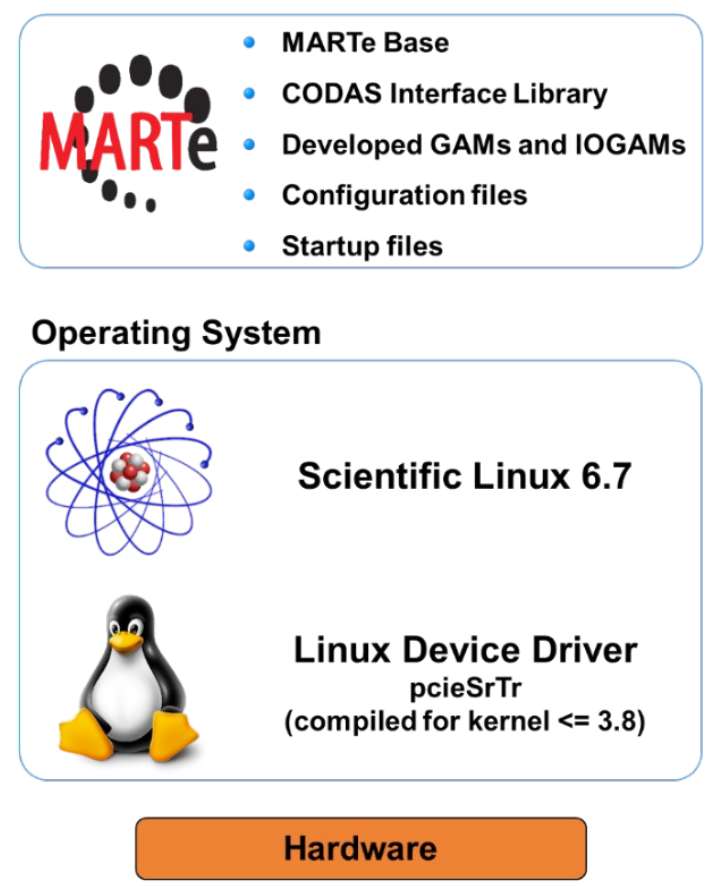

Fig. 3 - Software Architecture

\section{JET CODAS Integration}

The CODAS Interface Library provides features to connect MARTe to JET CODAS, enabling MARTe to receive/reply messages from/to JET CODAS and send the diagnostic data files. Fig. 4 depicts the coupling between MARTe and JET CODAS, showing the CODAS messages and the steps of a JET Pulse/MARTe Cycle:

(1) MARTe is initialized with a base configuration but, before the JET Pulse, it receives the configurations from the pulse schedule editor (Level-1) and sets up the acquisition boards.

(2) MARTe receives the trigger to start acquisition and starts acquiring to the board memory.

(3) During the JET Pulse, MARTe acquires data to the board memory.

(4) MARTe receives from CODAS the end pulse command and stops the acquisition.

(5) After the pulse, threads are launched to get the data from the board memory and to store the retrieved data in the host hard drive. To make the system stable, the threads (one per channel) run sequentially, avoiding out of memory crashes.

(6) Several minutes after the end of pulse, CODAS retrieves the data from local HDD to JET Data Archiver. 


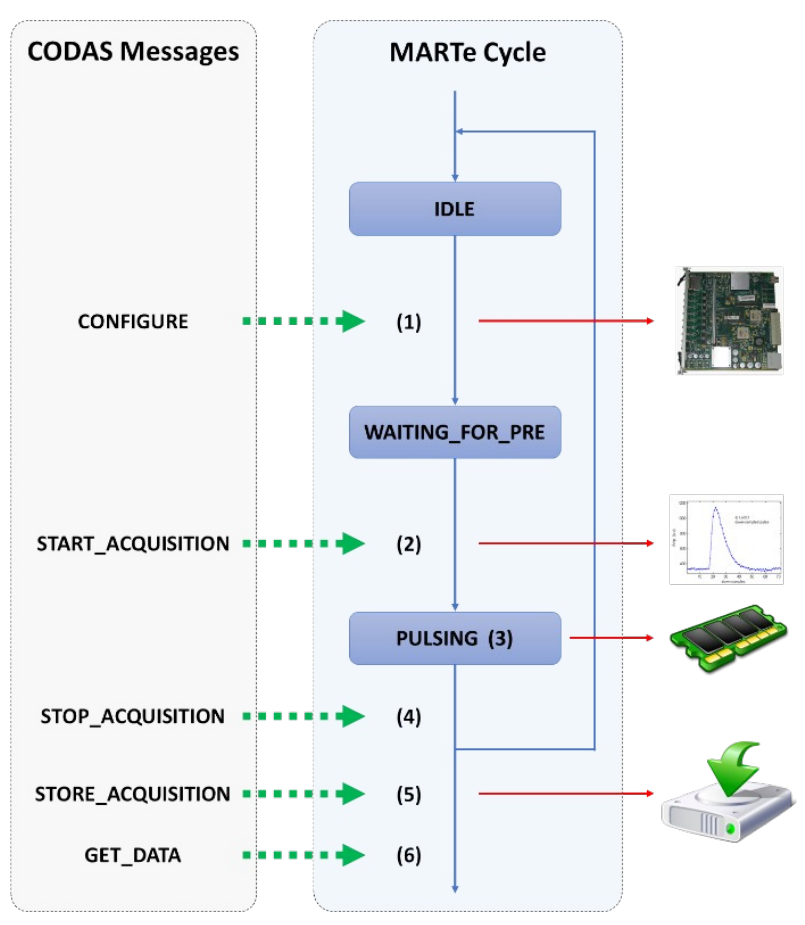

Fig. 4 - MARTe Integration with JET CODAS

\section{$5 \quad$ File mirroring}

During the uniformization process, a hierarchically organized directory structure that is common to all diagnostics was designed. Using this approach, the configurations can be mirrored between the other diagnostics, which contributes to make all the diagnostic hard-drives compatible, reducing the maintenance expertise.

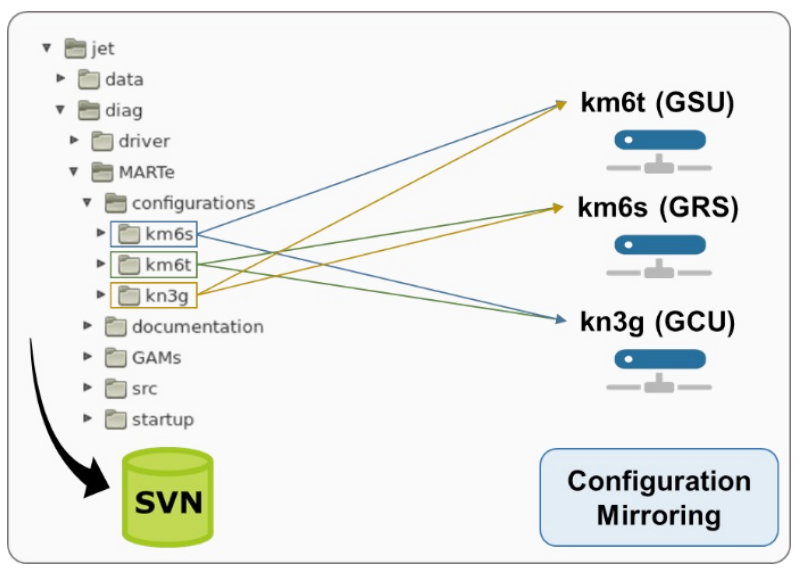

Fig. 5 - Directory Structure

Fig. 5 depicts the directory structured under the project root directory (jet). The installed software in all diagnostics is the same, except for the configurations directory in which the MARTe configuration files, specific for each diagnostic, are stored. Using file mirroring of these directories, a hard drive from one diagnostic can be used in any other with simple startup configurations to change the active diagnostic.

In addition, during the development phase all code was synchronized with svn [26], enabling a fast installation of a new diagnostic. If any change is done in the structure, or in versioned files, it can be easily synchronized with other diagnostics using the svn features.

\section{Conclusions}

This solution was designed to standardize the hardware and software of the three diagnostics:

- The usage of the same hardware may minimize the number of spare modules and reduces the device driver maintenance expertise.

- The software standardization minimizes the system specific expertise and can reduce the data backup devices. The provided file mirroring between systems enables the usage of a hard drive from one diagnostic to any other. In few steps, the configured startup diagnostic can be changed. The replacement of a malfunctional hard drive can be done using a backup from other diagnostic, avoiding the maintenance of 3 diagnostic backups.

The tests show that MARTe environment is a valuable and stable framework to manage diagnostics with post pulse data transfer like the presented ones. The data is stored to the board memory during the pulse and at the end is copied to the host and to the network archiver.

The presented solution was successfully tested for Scientific Linux 32 and 64 bits and is fully and stable running from JET Pulse \#91137@20/07/2016.

In the future, new added functionalities and changes in the core code will be synchronized with three diagnostics, contributing to maintain all the systems up to date and enables automatic code reutilization from one diagnostic in any other.

\section{Acknowledgements}

This work has been carried out within the framework of the EUROfusion Consortium and has received funding from the Euratom research and training programme 20142018 under grant agreement No 633053. IST activities also received financial support from "Fundação para a Ciência e Tecnologia" through project UID/FIS/50010/2013. The views and opinions expressed herein do not necessarily reflect those of the European Commission. Moreover, Fusion for Energy cannot be held responsible for any use which may be made of the information contained therein.

\section{References}

[1] V. G. Kiptily, F. E. Cecil, and S. Medley, Gamma-ray diagnostics of high temperature magnetically confined fusion plasmas, Plasma Phys. Control Fusion, 48 (2006), R59-R82.

[2] I. Zychor et al., High performance detectors for upgraded gamma ray diagnostics for JET DT campaigns, Phys. Scr. 91 (2016) 64003-64011

[3] R. C. Pereira et al., Pulse analysis for gamma-ray diagnostics ATCA sub-systems of JET tokamak, IEEE Trans. Nucl. Sci., 58 (4) (2011), 1531-1537

[4] A. M. Fernandes et al., Parallel processing method for high-speed real time digital pulse processing for gamma- 
ray spectroscopy, Fusion Eng. Des., 85 (2010), 308-312.

[5] R.C. Pereira et al., ATCA Fast Data Acquisition and Processing System for JET Gamma-Ray Cameras Upgrade Diagnostic, IEEE Trans. Nucl. Sci., 57 (2010), 683-687.

[6] A. M. Fernandes et al., Real-Time Processing System for the JET Hard X-Ray and Gamma-Ray Profile Monitor Enhancement, IEEE Trans. Nucl. Sci., 61(3) (2014), 12091215.

[7] R. Kwiatkowski, et al., CeBr3-based detector for gammaray spectrometer upgrade at JET, Fusion Eng. Des. (2017), http://dx.doi.org/10.1016/j.fusengdes.2017.02.103

[8] M. Curuia, et al., Upgrade of the tangential gamma-ray spectrometer beam-line for JET DT experiments, Fusion Eng. Des. (2017), http://dx.doi.org/10.1016/j.fusengdes.2017.05.064

[9] AdvancedTCA ${ }^{\circledR}$ Base Specification, Revision 3.0, March 24, 2008.

[10] J. Y. Journeaux et al., Plant Control Design Handbook (ITER 27LH2V), v 7.0 (2013).

[11] P. Makijarvi et al., Guideline for Fast Controllers, I/O Bus Systems and Communication Methods between Interconnected Systems (ITER 333K4C), v 2.0 (2013).

[12] R.C. Pereira, et al., ATCA data acquisition system for gamma ray spectrometry, Fusion Eng. Des. 83 (2008), 341-345.

[13] A.J.N. Batista, et al., ATCA Control System Hardware for the Plasma Vertical Stabilization in the JET Tokamak, IEEE Transactions on Nuclear Science, 57 (2010), 583588.

[14] PCI Express ${ }^{\circledR}$ for AdvancedTCA ${ }^{\circledR}$, Revision 3.4, May 21, 2003.

[15] A. Neto et al., FireSignal-Data acquisition and control system software, Fusion Eng. Des., 82 (2007) 1359-1364.

[16] A. Neto et al., The control and data acquisition software for the gamma-ray spectroscopy ATCA sub-systems of the JET-EP2 enhancements, Fusion Eng. Des., 83 (2008) 346349.

[17] A. Neto et al., Linux real-time framework for fusion devices, Fusion Eng. Des. 84 (2009) 1408-1411.

[18] A. Neto et al., MARTe: A Multiplatform Real-Time Framework, IEEE Trans. Nucl. Sci., 57 (2010), 479-486.

[19] A. Neto et al., MARTe Framework: a Middleware for RealTime Applications Development, Proceedings of ICALEPCS2011, Grenoble, Franc.

[20] B. Gonçalves et al., ITER fast plant system controller prototype based on ATCA Platform, Fusion Eng. and Des. 87 (2012) 2024-2029.

[21] B.B. Carvalho et al., The ITER Fast Plant System Controller ATCA prototype Real-Time Software Architecture, Fusion Eng. and Des 88 (6-8) (2013) 541-546.

[22] D. Valcárcel et al., Parallel Task Management Library for MARTe, IEEE Trans. Nucl. Sci., 61(3) (2014), 1222-1227.

[23] S. Yun et al., A shared memory based interface of MARTe with EPICS for real-time applications, Fusion Eng. and Des. 89 (2014) 614-617.

[24] C. Rapson et al. Coupling DCS and MARTe: two real-time control frameworks in collaboration, Fusion Eng. and Des., 89 (2014), 3125-3130.
[25] C. Piron, et al., Integration of the state observer RAPTOR in the real-time MARTe framework at RFX-mod, Fusion Eng. Des.

http://dx.doi.org/10.1016/j.fusengdes.2017.02.093

[26] Subversion website: https://subversion.apache.org/ 